\title{
3D magnetization transfer (MT) for the visualization of cardiac free-running Purkinje fibers: an ex vivo proof of concept
}

\author{
Julie Magat ${ }^{1,2} \circledast$ Arnaud Fouillet $^{1,2} \cdot$ Marion Constantin $^{1,2} \cdot$ Kylian Haliot $^{1,2} \cdot$ Jérôme Naulin $^{1,2} \cdot$ \\ Dounia El Hamrani ${ }^{1,2}$. David Benoist ${ }^{1,2} \cdot$ Sabine Charron $^{1,2} \cdot$ Richard Walton $^{1,2} \cdot$ Olivier Bernus $^{1,2} \cdot$ Bruno Quesson $^{1,2}$
}

Received: 13 October 2020 / Revised: 2 December 2020 / Accepted: 22 December 2020 / Published online: 23 January 2021

(c) The Author(s) 2021

\begin{abstract}
Objectives We investigate the possibility to exploit high-field MRI to acquire 3D images of Purkinje network which plays a crucial role in cardiac function. Since Purkinje fibers (PF) have a distinct cellular structure and are surrounded by connective tissue, we investigated conventional contrast mechanisms along with the magnetization transfer (MT) imaging technique to improve image contrast between ventricular structures of differing macromolecular content.

Methods Three fixed porcine ventricular samples were used with free-running PFs on the endocardium. $T 1, T 2^{*}, T 2$, and $M 0$ were evaluated on 2D slices for each sample at $9.4 \mathrm{~T}$. MT parameters were optimized using hard pulses with different amplitudes, offset frequencies and durations. The cardiac structure was assessed through 2D and 3D T1w images with isotropic resolutions of $150 \mu \mathrm{m}$. Histology, immunofluorescence, and qPCR were performed to analyze collagen contents of cardiac tissue and PF.

Results An MT preparation module of $350 \mathrm{~ms}$ duration inserted into the sequence with a $B 1=10 \mu \mathrm{T}$ and frequency offset $=3000 \mathrm{~Hz}$ showed the best contrast, approximately 0.4 between PFs and myocardium. Magnetization transfer ratio (MTR) appeared higher in the cardiac tissue (MTR $=44.7 \pm 3.5 \%)$ than in the PFs $(\mathrm{MTR}=25.2 \pm 6.3 \%)$.

Discussion MT significantly improves contrast between PFs and ventricular myocardium and appears promising for imaging the 3D architecture of the Purkinje network.
\end{abstract}

Keywords Magnetization transfer · Cardiac conduction system $\cdot$ Free-running Purkinje fibers

\section{Abbreviations \\ PF Purkinje fibers \\ MT Magnetization transfer \\ MTR Magnetization transfer ratio}

\section{Introduction}

The specialized cardiac conduction system (CCS) in the ventricles enables rapid and near synchronous excitation of the ventricular myocardium. It is composed of an extensive branching network of Purkinje fibers (PF) ensuring rapid electrical conduction due to specific electrical properties and a fibrous structure surrounded by a collagen sheath [1, 2]. These PF differ from working myocardial cells in size, function, structure, and arrangement [3]. The Purkinje conduction network plays a crucial role in normal cardiac function, but it has also been implicated in arrhythmogenesis and sudden cardiac death $[4,5]$. 3D imaging of its architecture is, therefore, of high importance to better understand cardiac arrhythmia mechanisms and improve their diagnosis and treatment.

The most common experimental techniques to study the architecture of the cardiac electrical conduction system are based on destructive approaches such as histology [6]. Ink injections have also been used to follow PF ramification in

2 Centre de Recherche Cardio-Thoracique de Bordeaux Inserm, U1045, Université de Bordeaux, 33000 Bordeaux, France 
sheep until their termination within the myocardium, but do not allow for quantitative reconstructions [7]. Recently, $\mu$-computed tomography has been applied with a very high resolution of $18 \mu \mathrm{m}$ on rat heart [8] to follow the CCS. The same team published a 3D representation of the CCS in the intact human heart with an isotropic resolution of $73 \mu \mathrm{m}$ [9]. However, disadvantages of the X-ray-based techniques are the ionizing radiation, the ability of contrast enhancement to discriminate between different tissues is limited and the transfer of method from microCT to medical CT facilities is currently not available.

Alternatively, magnetic resonance imaging (MRI) is another approach for non-destructive 3D acquisition of tissue samples. Some studies have developed algorithms to track free-running PF from high-resolution images in rodent hearts with an isotropic resolution of $25 \mu \mathrm{m}$ [10-12]. Hwang et al. [13] demonstrated at $17.6 \mathrm{~T}$ using a fast gradient echo sequence an alteration with aging in rabbit hearts especially on free-running PF thickness.

Different MRI acquisitions can be exploited to improve contrast in biological tissues, depending on their proton density and relaxation times. However, all these MR approaches suffer from an obvious lack of contrast between CCS and cardiac muscle on ex vivo fixed samples at high field [14].

MT MRI [15] has been widely been used in neuroradiology to visualize myelin defects in multiple sclerosis [16], as well as to suppress background signals for angiography applications [17]. It consists of using a preparation module (generally with off-resonance pulses) that saturates a portion of the proton spectrum from macromolecules and reduces partially the signal from the water proton frequency through transfer of magnetization [18]. MT is more specific for assessing macromolecular contents than classical contrast MRI techniques ( $T 1, T 2$ and proton density weighted images). Several approaches using MT for collagen detection have been applied to evaluate fibrosis in liver [19], in kidney [20,21] and fibrosis in intestinal tissue [22]. However, MT has rarely been investigated for cardiac imaging; and only few studies have used MT in vivo with off-resonance pulses to increase contrast between blood and muscle [15] and between lesion sites (infarct, hypoxia and edema areas) and healthy cardiac tissue [23]. Interestingly, a recent study [24] applied MT preparation in vivo to assess myocardial infarct. They demonstrated a difference of contrast between scar areas and healthy tissues. They found a good correspondence between low MTR and hyperintense signal using LGE for the assessment of myocardial scars.

Several types of collagen (mainly type I and III) were reported to be present in the heart with different expression levels in myocardium and PF [2, 25]. Moreover, different MT ratios were measured depending on the type of collagen
[26], making this technique a potential candidate for increasing image contrast between PF and myocardium.

In this work, we first quantitatively analyzed the contrast between PF and cardiac muscle using conventional MRI techniques based on a difference of structure. In a second step, off-resonance MT was applied in 2D to assess MT parameters that optimize the contrast to present a proof of concept to distinguish PF from cardiac tissue. From these results, 3D high-resolution images were acquired with MT with the objective of visualizing the free-running PF in cardiac tissue samples.

\section{Methods}

\section{Ex vivo samples}

Fixed ex vivo cardiac samples $(n=3)$ were obtained from three $\sim 40 \mathrm{~kg}$ male pigs under general anesthesia. Hearts were removed by sternal thoracotomy and flushed with cold cardioplegic solution. This protocol was accepted by the Animal Research Ethics Committee (Comité d'Ethique en Expérimentation Animale de Bordeaux; CEEA50) in accordance with the European rules for animal experimentation (European legislation 2010/63/UE; 2010).

Left ventricular samples from the anterior side of the heart were chosen due to the natural abundance of freerunning fibers in this region. Samples were cut in the longitudinal direction (Fig. 1). Three samples were prepared immersed in $10 \%$ formaldehyde mixed with an MRI contrast agent $(0.2 \%$ of GD DOTA was added of the total formaldehyde volume): sample 1 with a dimension of $3 \times 4 \times 7 \mathrm{~cm}$, sample 2 with a dimension of $4 \times 4 \times 6 \mathrm{~cm}$ and sample 3 with a dimension of $3 \times 3 \times 5 \mathrm{~cm}$. Samples were continuously and mechanically agitated in their fixing solution during 2 days in a cold room and then stored in their fixing solution in the same cold room.

For MRI acquisition, samples were removed from the solution and were immersed into fluorinert (Invertec Performance Medicals, Saint Priest France), a perfluoropolyether, giving no ${ }^{1} \mathrm{H}$ MR signal and having similar magnetic susceptibility as tissue [27].

\section{MRI experiments}

\section{Magnet}

Experiments were performed at $9.4 \mathrm{~T}$ within a horizontal bore of $30 \mathrm{~cm}$ (Bruker BioSpin MRI, Ettlingen Germany) with BGA-20S gradient system (maximum strength of $300 \mathrm{mT} / \mathrm{m}$, slew rate of $1170 \mathrm{~T} / \mathrm{m} / \mathrm{s}$ ). A cylindrical singlecoil element coil (83 $\mathrm{mm}$ inner diameter, with a maximum of $28 \mathrm{~W}$ power deposit in continuous wave) operating in 

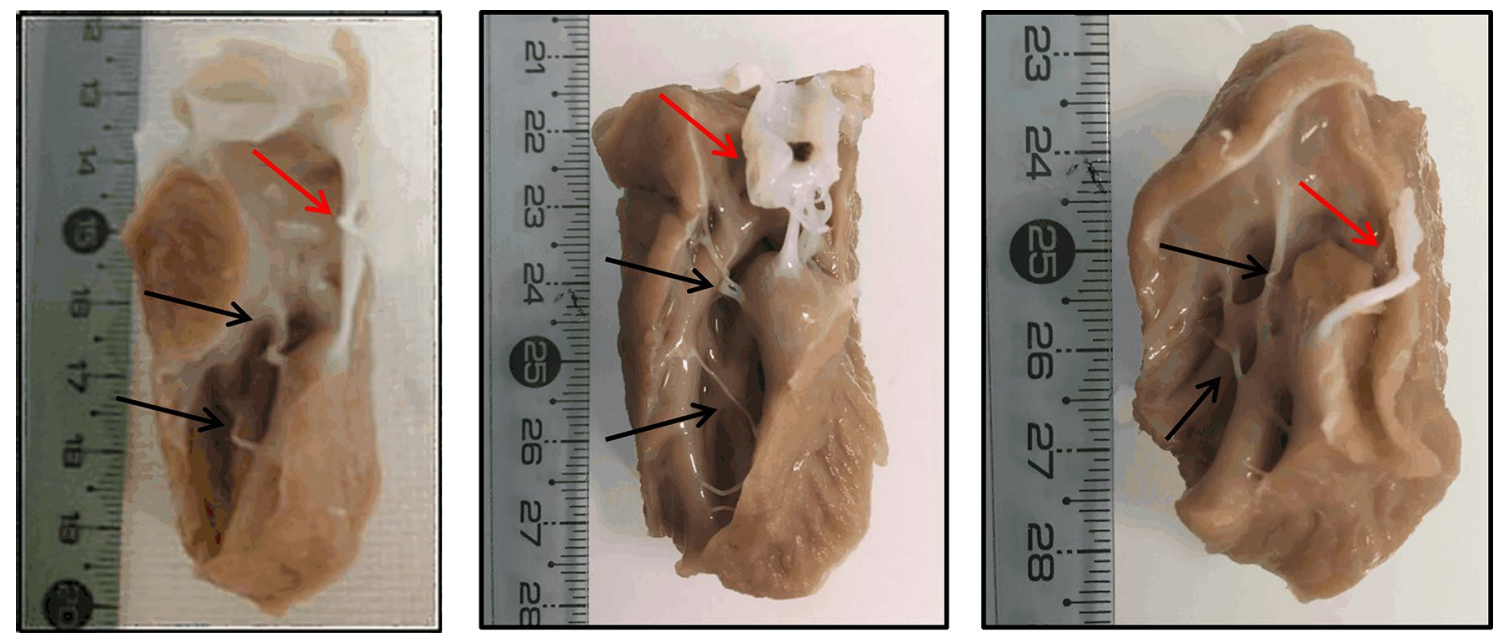

Fig. 1 Photographs of three ex vivo samples with contrast agent after fixation. Black arrows indicate free-running fibers of interest. Red arrows indicate tendons connected to valves

transmission and reception was used for fixed ex vivo imaging. A temperature probe (SA Instruments, Stony Brook, $\mathrm{NY}$ ) was placed on the top of the samples to record temperature during acquisitions.

\section{MRI sequences}

2D slice with a resolution of $200 \mu \mathrm{m} \times 200 \mu \mathrm{m} \times 1 \mathrm{~mm}$ using a FOV $=30 \times 30 \mathrm{~mm}$ and a matrix $=150 \times 150$ with a bandwidth of $75 \mathrm{kHz}$ was applied for $T 1, T 2, T 2 *$ and proton density $(M 0)$ imaging on samples.

A rapid acquisition with relaxation enhancement (RARE) sequence was used to determine $T 1$ relaxation time with a saturation recovery mode, where TR varied to collect series of $T 1$ weighted images. Imaging parameters were: $\mathrm{TE}=30.6 \mathrm{~ms}$, $\mathrm{TR}$ array $=200,400,800,1500,5000$ and $10,000 \mathrm{~ms}$, RARE factor $=8$ for a total acquisition time of $5 \min 22 \mathrm{~s}$.

A multi-echo spin echo sequence was used to determine $T 2$ relaxation time with $\mathrm{TR}=2000 \mathrm{~ms}, \mathrm{NA}=1,8$ echoes starting at $10 \mathrm{~ms}$ with increments of $10 \mathrm{~ms}$ for a total acquisition time of $5 \mathrm{~min}$.

A multi-gradient echo sequence with eight echos was launched to obtain $T 2 *$ information with $\mathrm{TR}=800 \mathrm{~ms}$, $\mathrm{NA}=1,8$ echoes starting at $4 \mathrm{~ms}$ with increments of $5.5 \mathrm{~ms}$ for a total acquisition time of $2 \mathrm{~min}$.

\section{D magnetization transfer (MT) imaging}

For each 2D acquisition, the sequence parameters were: $\mathrm{TE} / \mathrm{TR}=4 / 2000 \mathrm{~ms} ; \mathrm{FOV}=30 \times 30 \mathrm{~mm}$ matrix size $=150 \times 150 ; \mathrm{FA}=90^{\circ} ; \mathrm{NA}=2 ;$ a bandwidth of $50 \mathrm{kHz}$ for a total acquisition time of 10 min with a resolution of $200 \times 200 \times 500 \mu \mathrm{m}$. The MT module (hard pulse of amplitude $B 1$, offset frequency $\Delta f$ and module duration $D$ ) was added to the sequence before the excitation pulse of the FLASH sequence. One pulse lasts $5 \mathrm{~ms}$ with a bandwith of $256 \mathrm{~Hz}$. The following parameters were evaluated to study their influence on the contrast between fiber and muscle:

$$
\begin{aligned}
& \Delta f: 2000,3000,5000 \text { and } 10,000 \mathrm{~Hz} . \\
& \mathrm{B} 1: 5,10 \text { and } 15 \mu \mathrm{T} \text {. } \\
& D: 50,150,250,350,450 \mathrm{~ms} \text {. }
\end{aligned}
$$

\section{D magnetization transfer (MT) imaging}

The use of a contrast agent allows to decrease TR and consequently to increase the power deposit. FLASH acquisition using the MT module $(B 1=10 \mu \mathrm{T}, D=350 \mathrm{~ms}$ and $\Delta f=3000 \mathrm{~Hz})$ in $3 \mathrm{D}$ was acquired with $\mathrm{TR}=350 \mathrm{~ms}$, $\mathrm{TE}=4.3 \mathrm{~ms}, \mathrm{NA}=4, \mathrm{FA}=60^{\circ}$, Bandwidth of $50 \mathrm{kHz}$ and a resolution $=150 \times 150 \times 150 \mu \mathrm{m}^{3}$. For the first sample, a FOV $=35 \times 40 \times 35 \mathrm{~mm}$ using $466 \times 266 \times 233$ pixels, for a total acquisition time of $24 \mathrm{~h} 6 \mathrm{~min}$ is applied, for the second sample a FOV $=60 \times 40 \times 40 \mathrm{~mm}$ using $400 \times 266 \times 266$ pixels, for a total acquisition time of $27 \mathrm{~h} 30 \mathrm{~min}$ and for the third sample a FOV $=50 \times 30 \times 30 \mathrm{~mm}$ using $328 \times 200 \times 200$ pixels, for a total acquisition time of $15 \mathrm{~h} 30 \mathrm{~min}$ is applied.

\section{Data processing}

Images were exported and post-processed using a homemade program written in Matlab. $T 1, T 2 *, T 2$ of tissue and PF were obtained from exponential curve fitting on a pixel-bypixel basis. Two ROIs localized in the fiber and muscle were manually drawn, from which the mean and standard deviation of relaxation times were evaluated. $M 0$ was extracted on $T 2^{*}$ fitting using a nonlinear fit with three unknown variables. 
For each magnitude image sequence, three ROIs were manually drawn: (1) surrounding the free PF, (2) in the tissue (parenchyma) and (3) in a region considered as the background to assess noise. Manually ROIs in the cardiac tissue, fiber, and noise were drawn. Signal-to-noise ratio (SNR) for $\mathrm{PF}$ and tissue were computed as a mean of signal for voxels inside the ROI in the tissue of interest (muscle or fiber) divided by as the average standard deviation of voxel intensity obtained in ROI from the background region for the volume coil. A magnetization transfer ratio (MTR) was defined by $\mathrm{MTR}=100 *\left(S_{0}-S_{\mathrm{MT}}\right) / S_{0}$.

Where $S_{0}$ represents the signal without an MT module and $S_{\mathrm{MT}}$ is the signal with the MT module. The MT contrast between fiber and tissue was calculated as follow using contrast to noise ratio (CNR) defined by signal difference between fibers and the surrounding parenchyma divided by background noise:

$\mathrm{CNR}=2\left(\mathrm{SNR}_{f}-\mathrm{SNR}_{t}\right) /\left(\mathrm{SNR}_{f}+\mathrm{SNR}_{t}\right)$.

where $\mathrm{SNR}_{f}$ and $\mathrm{SNR}_{t}$ are the $\mathrm{SNR}$ of the fibers and parenchyma, respectively. Visualization of $3 \mathrm{D}$ volume rendering was obtained using Volview (Kitware, Clifton Park, NY). Free-running PF segmentation was performed using Snake Interaction Mode algorithm in 3D with itksnap software (http://www.itksnap.org).

\section{Histological analysis}

Histological analysis was performed to identify Purkinje fibers.

Volumes of $0.4 \times 1.5 \times 0.5 \mathrm{~cm}$ were obtained from the three samples. Sections were localized around an intact external "free-running" fiber. Each block of tissue was embedded in paraffin and sectioned at $6 \mu \mathrm{m}$ in the longitudinal fiber axis. Tissue sections were stained with Masson's trichrome for the identification of different structure: coloration in blue-green reveals collagen, cellular nuclei are stained in blue-black and myocytes in red. Photographs were taken on an optical microscope (Nikon Eclipse 80i, Nikon, Tokyo, Japan) equipped with a digital camera (Nikon Ds Fi2).

\section{Detection of collagen types I and III}

Additional analyses were performed to assess the relative contents of collagen I and III in purkinje fibers and cardiac myocytes, using immunofluorescence staining (qualitative imaging) and Reverse Transcription quantitative PCR (quantitative measure at the mRNA level).

\section{Immunofluorescence staining}

Frozen sections (10 $\mu \mathrm{m}$ thick) of ventricular myocardium and PFs taken from pig hearts $(N=3)$ were fixed into formalin (10\%). Following a blocking step (10\% normal goat serum, Uptima-Interchim UP379030), sections were incubated with primary antibodies rabbit polyclonal anticollagen I (Abcam: catalog no. ab34710; 1:100) or rabbit polyclonal anti-collagen III (Abcam: catalog no. ab37778; 1:100) overnight at $4{ }^{\circ} \mathrm{C}$. Sections were then washed in PBS and incubated during $1 \mathrm{~h}$ at room temperature with secondary antibodies Alexa Fluor 488 Chicken anti-rabbit (Invitrogen A21441; 1/200) or Alexa Fluor 555 Donkey anti-rabbit (Invitrogen A31572; 1/200). Nuclei were stained with DAPI (Invitrogen D1306, 1:100,000 in PBS).

Images were captured under an inverted fluorescence microscope (Nikon NiE) with DSRi2 camera (Nikon).

\section{Reverse transcription quantitative PCR (RT-qPCR)}

Samples (ventricular myocardium and PF) from three pig hearts were dissected out and immediately placed into RNA later Buffer. Biopsies were then stored at $4{ }^{\circ} \mathrm{C}$ until RNA extraction. Total RNA was extracted from tissues using QIAzol reagent (QIAGEN). RNA was purified and DNase treated using the QIAGEN RNeasy Kit (QIAGEN). RNA quantity was assessed by spectrophotometry (NanoDrop/ Thermofisher).

Sequences for primers were obtained from Ensembl Genome Browser. Primers were designed using Primer designing tool (NCBI) and synthesized at Sigma Aldrich/ Merck. 400 ng of RNA was reversed transcribed using a cDNA Reverse Transcription kit (BIO-RAD) according to the manufacturer's protocol. RT-qPCR was performed in a $10-\mu \mathrm{L}$ reaction volume $(1 \mu \mathrm{L}$ cDNA, $5 \mu \mathrm{L}$ of SYBR Green mix (BIO-RAD), a volume with a concentration of $10 \mu \mathrm{m}$ upstream and downstream primers respectively, and added ddH2O to $10 \mu \mathrm{L}$ on the BIO-RAD C100 Touch Thermal Cycler/CFX96 Real-time System. Expression levels for Colla1 (collagen type I) and Col3a1 (collagen type III) were normalized using a normalization factor calculated by CFX Manager software (BIO-RAD) and based on RT-qPCR results for two selected housekeeping genes, HPRT1 and GUSB.

\section{Statistical analysis of qPCR}

Data are presented as mean \pm SEM for three pigs. Two nonparametric tests were used ( $2 t$ test/Mann-Whitney): one to compare the response to collagen I between cardiac tissue and PF and one to compare the expression of collagen III between muscle and fiber. Samples were considered 
significantly different for $\mathrm{p}$ values lower than 0.05 . One asterisk (*) identifies $P$ values between 0.01 and 0.05 .

\section{Results}

\section{MR parameters}

The mean relaxation times ( $T 1, T 2$ and $T 2 *$ in milliseconds) and mean of $M 0$ (a.u) are listed in Table 1. $T 1$ in the fiber is equal to $458 \pm 42 \mathrm{~ms}$ and is quite similar with $T 1$ in the tissue $(428 \pm 21 \mathrm{~ms}) . T 2$ exhibits values to $16.0 \pm 1.8 \mathrm{~ms}$ and $18.1 \pm 0.4 \mathrm{~ms}$ in the tissue and the fiber, respectively. Note that $T 2$ was smaller in the tissue whereas $T 2 *$ values were similar in the muscle and the fiber for all samples with values closed to $11.5 \mathrm{~ms}$. Signals from $M 0$ appeared to be closed in the tissue and the fiber for all samples: $M 0=31.6 \pm 3.9$ a.u. and $M 0=31.1 \pm 3.2$ a.u. for cardiac tissue and fiber, respectively. From these data, very limited contrast can be obtained between fiber and tissue using conventional imaging techniques, justifying the need for alternative contrast enhancing approaches. Theoretically, no contrast can be evaluated for using FLASH sequence between fiber and cardiac tissue with these relaxations times and $M 0$ values.

\section{MT optimization}

Without MT (Fig. 2a), a nearly homogeneous signal intensity was observed (proton density weighted sequence) for the first sample. In presence of MT, the contrast is improved and more structural details can be identified (Fig. 2b).

CNR was calculated for each sample using a region of interest in the tissue and a region of interest along fibers (red region of interests in Fig. 2a). Graphs on Fig. 2 represents CNR mean for cardiac samples without and with MT as function of $\Delta \mathrm{f}(2,3,5$ and $10 \mathrm{kHz}), B 1(5,10,15 \mu \mathrm{T})$ and $\mathrm{D}$ $(50,150,250,350$ and $450 \mathrm{~ms})$.

The contrast value without MT (equal to $0.06 \pm 0.03$ ) is plotted at a zero offset frequency on each graph. Whatever the MT pulse parameters, contrast was systematically higher with the preparation module. However, contrast curves show different values depending on the MT parameters: maximal values for the contrast between tissue and fibers were found at a frequency offset of $2-4 \mathrm{kHz}$ (red ellipses on Fig. 2c), for an amplitude of $10 \mu \mathrm{T}$ applied during $350 \mathrm{~ms}$ (maximal value of 0.52 ) or for an amplitude of $15 \mu \mathrm{T}$ applied during $450 \mathrm{~ms}$ (maximal value of 0.65 ).

For $B 1=10 \mu \mathrm{T}$ and $B 1=15 \mu \mathrm{T}$, temperature was monitored with a raise of temperature during 2 min and a plateau of maximal value until the end of acquisitions. The mean of the temperatures during the plateau is reported in Fig. 3 during 2D acquisition with MT module as a function of $B 1(10$ and $15 \mu \mathrm{T})$ and $\mathrm{D}(50,150,250,350$ and

$450 \mathrm{~ms}$ ) at the same repetition time $\mathrm{TR}=2 \mathrm{~s}$. No temperature increase is measured for $B 1=5 \mu \mathrm{T}$ at different module duration. Histograms present an increase of temperature below $2{ }^{\circ} \mathrm{C}$ for $B 1=10 \mu \mathrm{T}$ and $D \leq 350 \mathrm{~ms}$. When $B 1=15 \mu \mathrm{T}$ and $D \geq 350 \mathrm{~ms}$, the temperature is above $4{ }^{\circ} \mathrm{C}$ and reaches a maximum value of $5.8^{\circ} \mathrm{C}$ with $B 1=15 \mu \mathrm{T}$ and $D=450 \mathrm{~ms}$. MT parameters is chosen with $B 1=10 \mu \mathrm{T}, D=350 \mathrm{~ms}$ and $\Delta f=3 \mathrm{kHz}$ with minimum effect of temperature (less than $2{ }^{\circ} \mathrm{C}$ ) and good contrast between fiber and cardiac tissue for $2 \mathrm{D}$ acquisitions.

\section{MT characterization}

Figure 4 displays 2D MR images without MT module, with MT module and MTR maps (Fig. 4a-c) for sample 1 (first line) sample 2 (second line) and sample 3 (third line). Figure $4 \mathrm{~b}$ shows that the signal is greater for the external fiber and several discrete structures within the muscle, whereas the bulk of the myocardium showed an attenuation of signal. MT effects are visualized by the MT ratio in Fig. $4 c$, for the fiber the MTR appears to lower than in the tissue.

Table 2 displays mean of SNR and mean of MTR from the fiber and the tissue for all three samples (extracted for regions of interest defined by red ROI on Fig. 4a), together with mean CNR. Under these conditions, SNR from tissue and fibers without MT preparation are similar, reaching approximately $71.6 \pm 18.4$ and $77.2 \pm 18.6$. We notice that the SNR in the fiber is always higher ( $45 \%$ of increase) than in the cardiac muscle with a MT module. Moreover, MT is more effective in the muscle: MTR $=44.7 \%$ in the tissue whereas MTR $=25.2 \%$ for the fiber (see Table 2). Nevertheless, the CNR between myocardium and fibers calculated is studied with a contrast close to 0.38 in mean for all samples.

\section{D MT visualization}

Figure 5 presents $2 \mathrm{D}$ views extracted from $3 \mathrm{D}$ acquisition with $\mathrm{TR}=350 \mathrm{~ms}$ for the three samples with identical MT parameters $(\Delta f=3 \mathrm{kHz}, B 1=10 \mu \mathrm{T}$ and $D=350 \mathrm{~ms})$. A first transverse slice is presented for the three samples (Fig. 5a), zooms corresponding to the red square highlight the structure at the endocardial insertion site of fibers (Fig. 5b). A $3 \mathrm{D}$ volume rendering for the three samples is presented, two free-running fibers are segmented for each piece in red (Fig. 5c) at a resolution of $150 \mu \mathrm{m}$.

Table 1 Mean of MR parameters $T 1, T 2$ and $T 2 *(\mathrm{~ms})$ are calculated and $M 0$ (a.u) measured for both cardiac tissue and fiber

\begin{tabular}{lll}
\hline & Tissue & Fiber \\
\hline $\mathrm{T} 1(\mathrm{~ms})$ & $458 \pm 42$ & $428 \pm 21$ \\
$\mathrm{~T} 2(\mathrm{~ms})$ & $16.0 \pm 1.8$ & $18.1 \pm 0.4$ \\
$\mathrm{~T} 2 *(\mathrm{~ms})$ & $11.3 \pm 2.5$ & $11.6 \pm 2.1$ \\
$\mathrm{M} 0$ (a.u.) & $31.6 \pm 3.9$ & $31.1 \pm 3.2$ \\
\hline
\end{tabular}




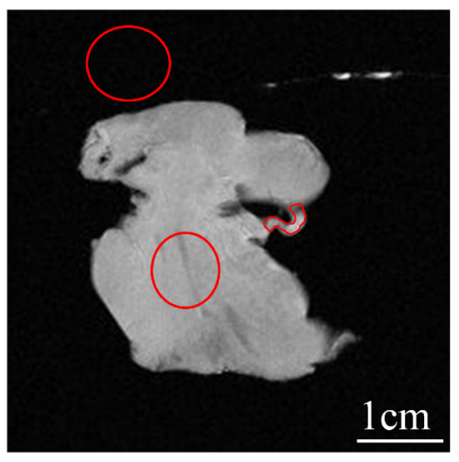

a)

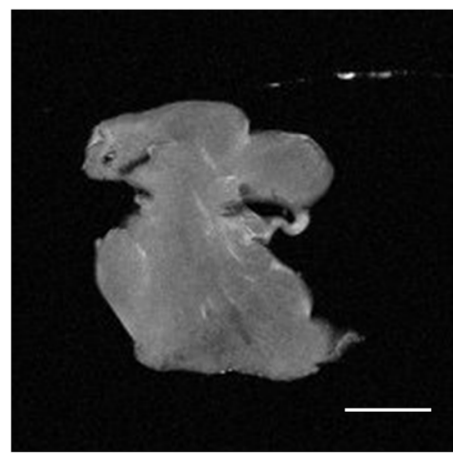

b)
$B 1=5 \mu T$

c)

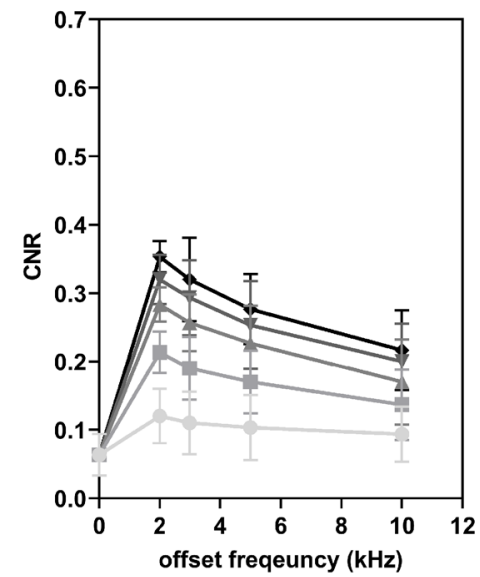

$B 1=10 \mu T$

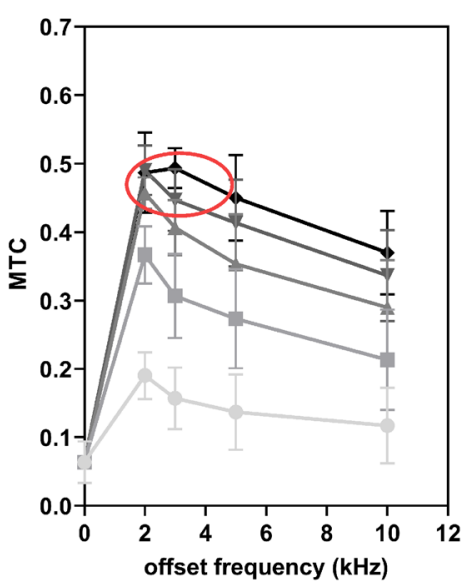

$B 1=15 \mu \mathrm{T}$

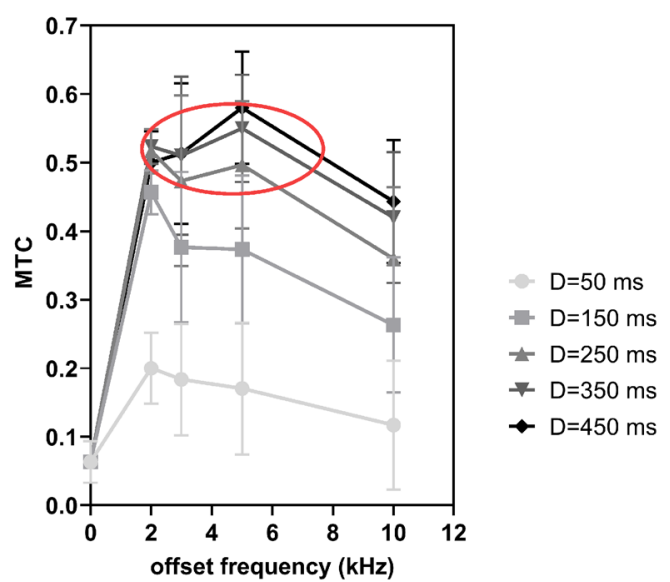

Fig. 2 2D MR images without (a) and with (b) MT $(\Delta f=5 \mathrm{kHz}$, $B 1=10 \mu \mathrm{T}, D=350 \mathrm{~ms}$ ). c Comparison of contrast of magnetization (CNR) in function of the offset frequency $(2,3,5$ and $10 \mathrm{kHz})$ at various $B 1$ amplitude $(5,10,15 \mu \mathrm{T})$ and MT module duration $(50,150$, 250,350 and $450 \mathrm{~ms}$ ). The contrast without MT is displayed at zero

\section{Histology results}

Figure 6a and b show typical macroscopic and a histological view, respectively, of the first sample following Masson's trichrome coloration. Figure $6 \mathrm{c}-\mathrm{e}$ display zooms to different areas containing PF (black circles in Fig. 6b). A blue-green coloration identifies collagen (yellow arrows) whereas cardiomyocytes appear in pink (red arrows). Figure $6 \mathrm{c}$ and d delineate free-running fibers surrounded by a collagen sheath in blue-green. Figure 6e displays insertion of fiber inside the myocardium. The thickness of fibers was found to be $600 \mu \mathrm{m}$ and $200 \mu \mathrm{m}$, respectively. External fibers located close to cardiac muscle displayed a thickness of around $\sim 180 \mu \mathrm{m}$. It is noted that the thickness of intramural Purkinje fibers is considerably reduced, down to $50-80 \mu \mathrm{m}$, and corresponding with the presence of collagen fibers within the cardiac muscle. offset frequency. Maximal values for the contrast between tissue and fibers were found at frequency offset of $3 \mathrm{kHz}$, for amplitude of $10 \mu \mathrm{T}$ applied during $350 \mathrm{~ms}$ and at frequency offset of $5 \mathrm{kHz}$, for amplitude of $15 \mu \mathrm{T}$ applied during $450 \mathrm{~ms}$ (red ellipses)

\section{Detection of collagen I and collagen III}

Figure 7 a displays illustrative examples resulting from immunofluorescence staining to show response to collagen I and III on PF and cardiac tissues. No difference in intensity for collagen I (in red) is observed between purkinje cells and cardio myocytes whereas for collagen III a more pronounced intensity (in green) is observed for purkinje cells than for cardiomyocytes. Staining with DAPI confirmed presence of cells in blue in tissues for all immunofluorescence images.

Graph bars (in Fig. 7b) present q-PCR expression results for cardiac tissue and PF on collagen I and collagen III. For collagen I, mean values of $1.28 \pm 0.16$ and $0.92 \pm 0.35$ are calculated for cardiac tissue and purkinje fibers, respectively, without statistical significant difference. For collagen III, mean values of $0.011 \pm 0.004$ and $2.12 \pm 1.19$ were calculated for cardiac tissue and purkinje fibers, respectively, 

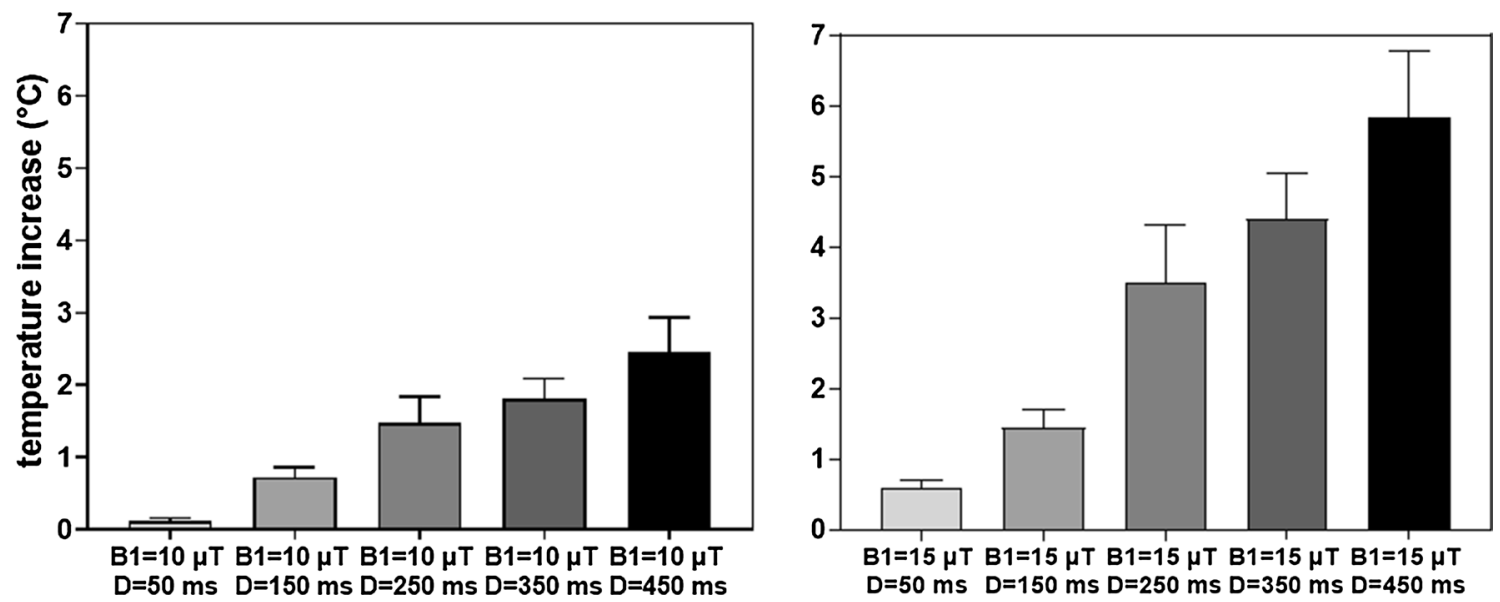

Fig. 3 Maximal temperatures raise measured during 2D acquisition with MT modules applied. Histograms represent temperature as a function of $B 1=10 \mu \mathrm{T}$ w for the first graph and $B 1=15 \mu \mathrm{T}$ for the second graph combined with MT module duration of 50, 150, 250, 350 and $450 \mathrm{~ms}$

resulting in a significant difference $(p=0.044)$ between both structures.

\section{Discussion}

In this work, we studied MRI parameters at high field (9.4 T) to visualize in $3 \mathrm{D}$ the PF network in the heart from large mammalian species. We showed that conventional contrast mechanisms relying on relaxation parameters or proton density result in limited contrasts between PF and myocardium. Relaxation times measured here are similar to those reported in previous studies at $9.4 \mathrm{~T}$ on fixed ex vivo rat heart [27-30]. An alternative approach was implemented to improve contrast between PF and myocardium, using MT. Both 2D and 3D acquisitions from three pigs hearts were successfully imaged with high correspondence to collagensheathed Purkinje fibers identified by histology.

With MT imaging, we were able to segment PF from cardiac tissue and tendons (red arrow in Fig. 1), whereas very limited contrast $(\mathrm{CNR}=0.03 \pm 0.01)$ could be observed between tendons and cardiac tissue (image not shown). We showed that MTR is non-zero for both tissue and fibers. However, a greater sensitivity in myocardium provided contrast with fibers. Since the effect is stronger in tissue, this technique is suitable to increase its contrast relative to fiber. The optimal contrast over the investigated experimental conditions was found at $5 \mathrm{kHz}$ for the highest $B 1$ value $(15 \mu \mathrm{T})$ applied during $450 \mathrm{~ms}$. However, the associated temperature rise was excessive (higher than $3{ }^{\circ} \mathrm{C}$ and up to $6{ }^{\circ} \mathrm{C}$ ) under these experimental conditions. Selecting lower energy deposition by setting a $B 1$ of $10 \mu \mathrm{T}$ at around $3 \mathrm{kHz}$ during $350 \mathrm{~ms}$ resulted in similar contrast with much lower temperature increase (less than $2{ }^{\circ} \mathrm{C}$ ).

\section{MT and gadolinium contrast agent}

In our experiments, we chose to perfuse the heart sample with gadolinium during the fixation process to reduce $T 1$ saturation effects. $0.2 \%$ of GD Dota was added in the perfusion preparation of samples. This contrast agent dose is a good compromise to obtain an increase of signal-to-noise ratio by decreasing $T 1$ values without increasing susceptibility effects on fixed tissue at $9.4 \mathrm{~T}$ [31]. In this study, $T 1, T 2$, $T 2^{*}$ and $M 0$ are similar in the conductive fibers and cardiac tissue on samples with contrast agent, we did not observe a difference of relaxation times and contrast (see Table 1), gadolinium is considered uniformly distributed during the fixation process. Same limited contrast was observed in figure S2 on ex vivo cardiac sample using MT MRI without contrast agent preparation [14]. Interestingly, Jones et al.[32] demonstrated on ex vivo hearts that the presence of a paramagnetic contrast agent increases contrast between ischemic and non-ischemic tissue with MT preparation. This was also observed in oncology: contrast agent administration during acquisition combine with MT preparation intensifies contrast when this difference of contrast is already present without MT [33, 34]. Our results with MT are in agreement with those from recent studies using MT to target collagen within fibrosis, such as for imaging progression of renal fibrosis in mice at 16.4 T [20]. The same team applied the MT method on swine kidney in vivo at $3 \mathrm{~T}$ with identical methodology, which was further validated using histology [21].

As a result, shorter repetition times could be applied, leading to shorter acquisition duration while preserving high signal-to-noise ratios ( $>40)$ at a $150 \mu \mathrm{m}$ isotropic spatial resolution whatever the experimental conditions. 
(a)
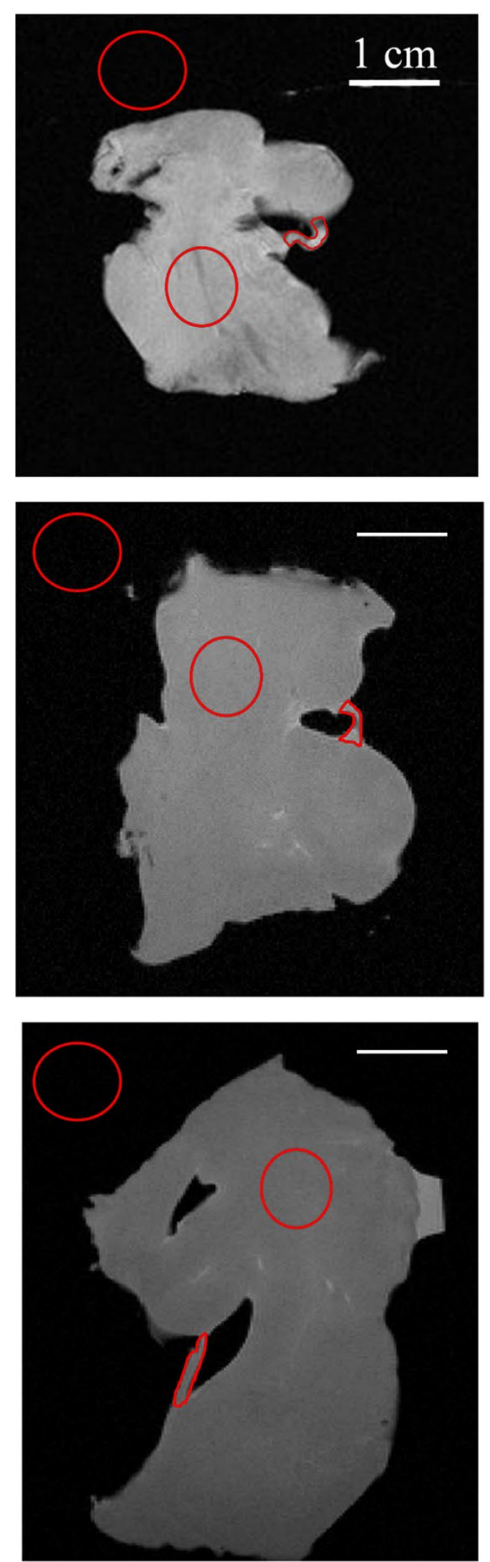

(b)
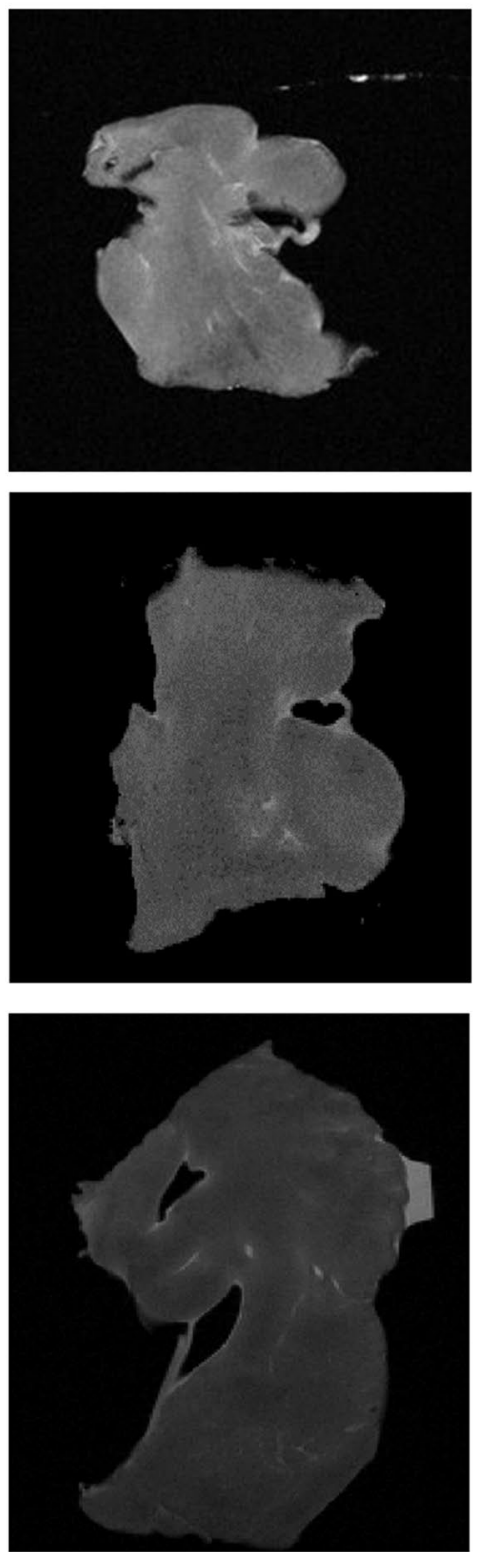

(c)
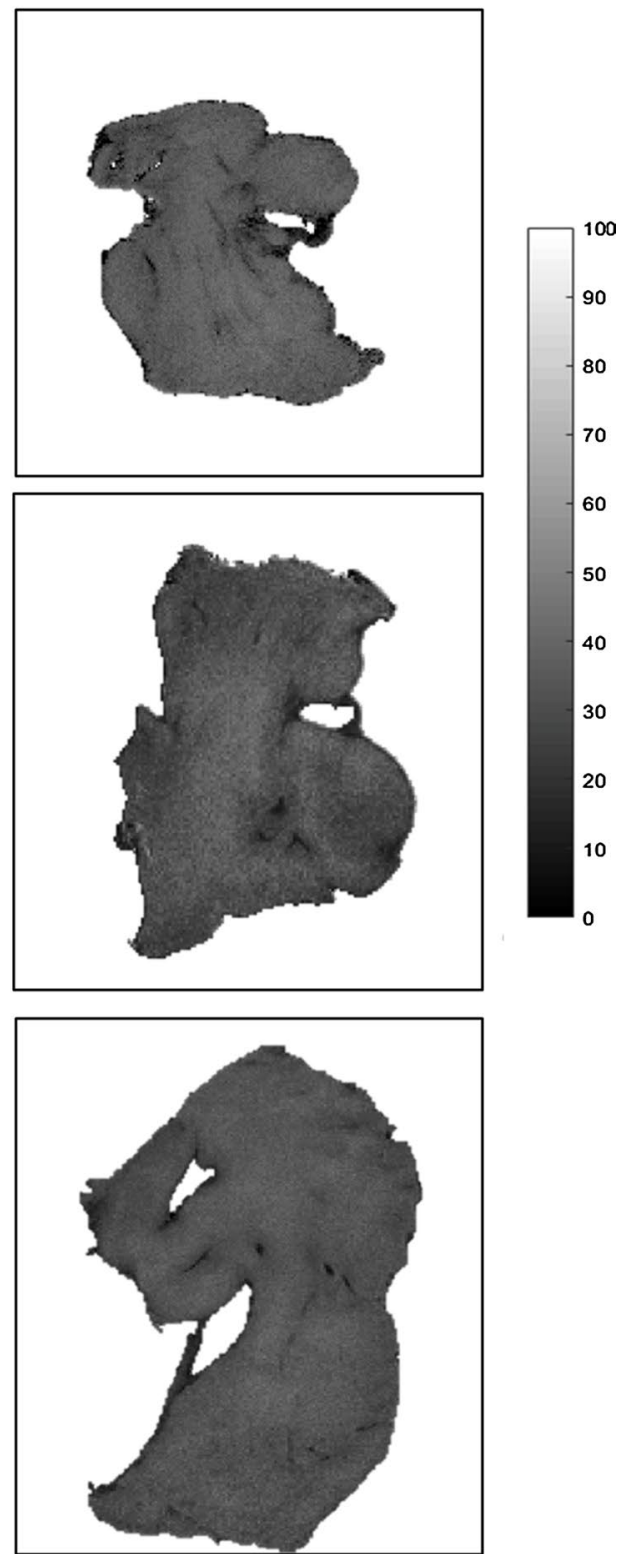

Fig. 4 2D images obtained a without the preparation module and b using MT for sample 1 (first line), sample 2 (second line) and sample 3 (third line). c MTR ratio is evaluated between 0 and $100 \%$

Table 2 Mean of SNR, CNR, and MTR (\%) on 2D image slices of the tissue and fiber under the same acquisition parameters

\begin{tabular}{llllll}
\hline & SNR fiber & SNR tissue & CNR & MTR fiber (\%) & MTR tissue (\%) \\
\hline Without MT & $77.2 \pm 18.6$ & $71.6 \pm 18.4$ & $0.06 \pm 0.03$ & - & - \\
MT & $58.4 \pm 16.5$ & $40.1 \pm 12.4$ & $0.38 \pm 0.8$ & $25.2 \pm 6.3$ & $44.7 \pm 3.5$ \\
\hline
\end{tabular}


(a)
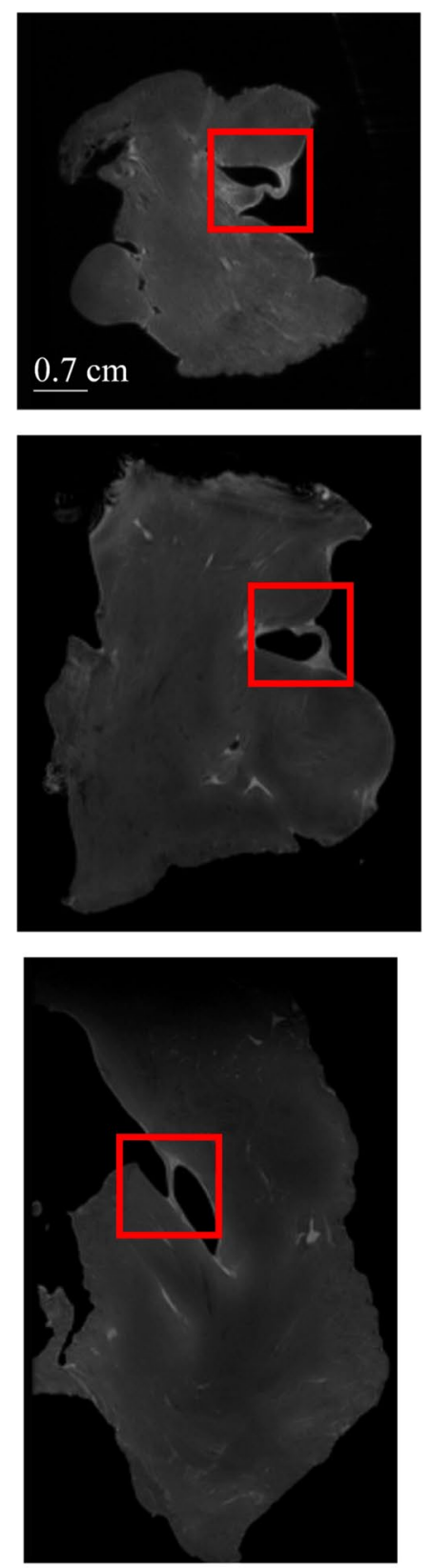

(b)
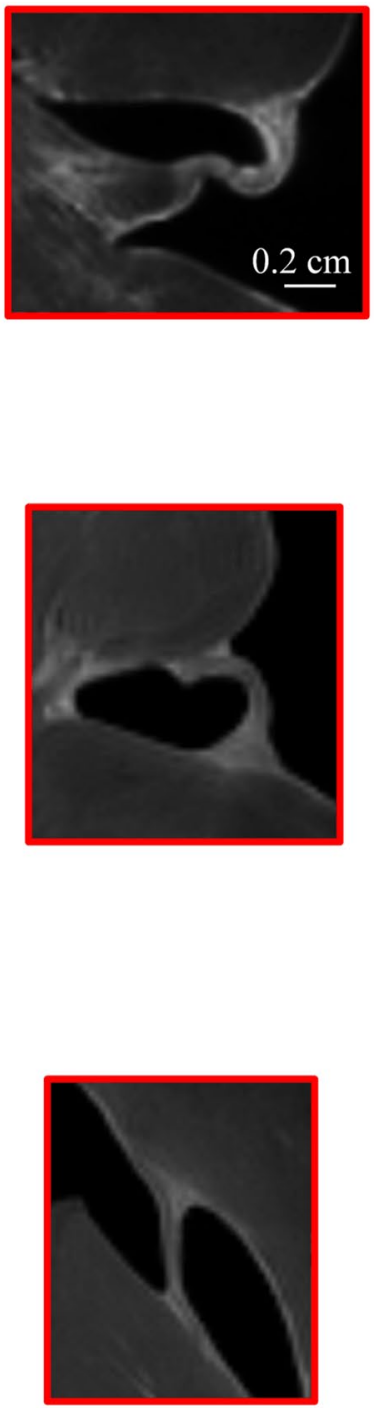

(c)
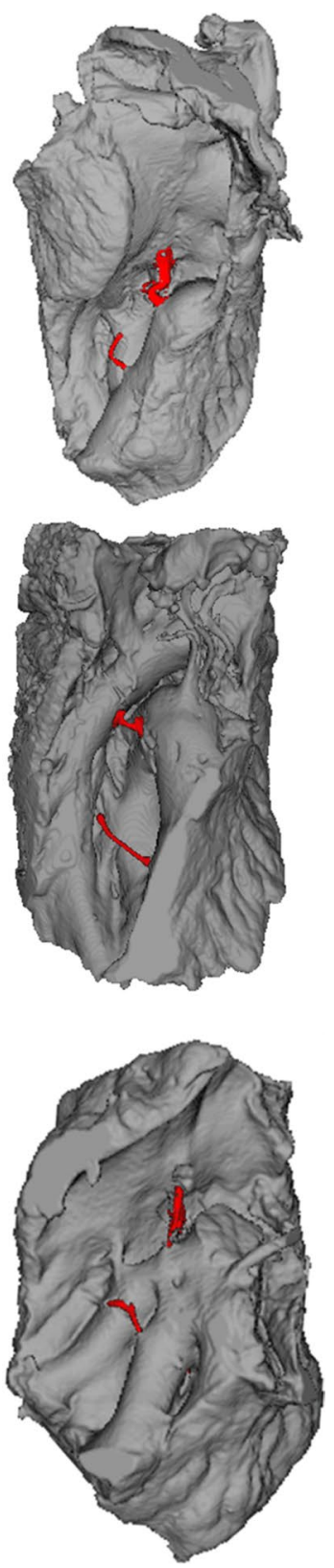

Fig. 5 a Transverse slices orientated from the 3D volume at an isotropic resolution of $150 \mu \mathrm{m}$ using MT MRI for sample 1, 2 and 3 . Insertion points of the fiber into the myocardium are expanded in the

\section{MT and temperature effect}

MT protocol using off-resonance pulses requires long acquisition times to deliver sufficient energy to obtain the desired image contrast. However, assuming an acceptable SNR limit of 25 for exploitable image analysis, the number of averages in 3D acquisition could be divided by 4 and consequently decrease the acquisition time further. The repetition red box and zoomed (b). c Fibers are segmented in red on a 3D volume rendering using ItkSnap

time was deliberately chosen with long intervals $(>4 T 1)$ to selectively emphasize the effect of MT, with a negligible $T 1$ contrast (less than $1 \%$ with $\mathrm{Gd}$ ).

In our $2 \mathrm{D}$ acquisition protocol, MT pulses resulted in an accumulated energy deposition of $2.2 \pm 0.5 \mathrm{~W} / \mathrm{TR}$ and non-negligible temperature increase. In $3 \mathrm{D}$, a shorter TR was selected to avoid excessive acquisition durations, but at the cost of an increase of energy deposition of $6.5 \pm 1 \mathrm{~W} /$ 


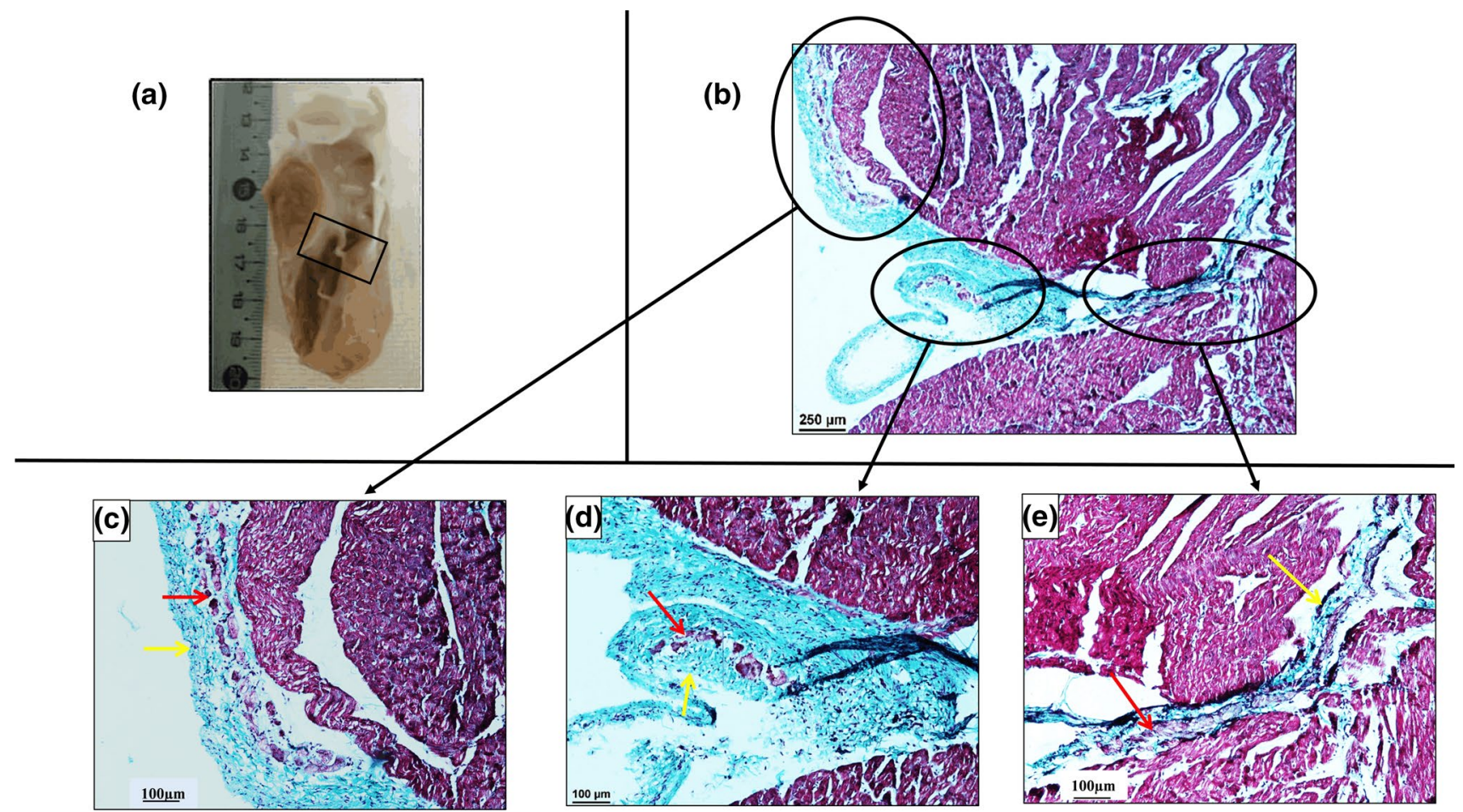

Fig. 6 Histology using Masson's Trichrome stain for a piece of sample 1 (a). Microscopy image (b) along purkinje fiber. c Zoom on different area corresponding to first black circle. d Second black circle,

TR. Although this value was acceptable to avoid MR coil alteration (maximal allowed energy deposition of $28 \mathrm{~W} /$ TR), the increase in temperature was around $5{ }^{\circ} \mathrm{C}$, which was considered at no risk for tissue integrity since the absolute temperature never exceeded $24{ }^{\circ} \mathrm{C}$. Although $T 1$ varies with temperature [35], these variations remain modest and of the order of $1 \%$ for an increase of temperature of $1{ }^{\circ} \mathrm{C}$ (see Figure $\mathrm{S} 1$ in supplementary materials). The effect on the observed contrast here cannot be considered prominent since the flip angle of the sequence was adjusted at the Ernst angle for repetition times of $350 \mathrm{~ms}$ (in 3D) and $2000 \mathrm{~ms}$ (in 2D) at the beginning of the acquisition.

\section{MT and collagen content}

A decrease in SNR was expected using MT since extracellular scaffold proteins (typically collagen) are present in almost every compartment of cardiac muscle. Yet, the epimysial layer of the extracellular matrix surrounding PF is extensive, particularly in free-running fibers. Consequently, MTR provided differential signal between myocardial tissue and PF-containing regions, resulting in increased contrast. Such a difference could be further related to a variable ratio of collagen types between cardiac muscle and PF. Indeed, we demonstrated with immunofluorescence an intense response to collagen III in PF in comparison to myocardium and we (e) and third black circle. In blue the collagen (yellow arrow), in pink the cardiomyocytes (red arrow)

observed a similar response to collagen I for cardiac tissue and conductive fibers. These observations were validated quantitatively with RT-qPCR, showing an intense positive reaction of collagen III in fibers in comparison to cardiac tissue. These results are in good agreement those reported in the literature for the myocardium, where type I collagen was reported to be the most abundant [25], whereas immunochemistry performed on sheep cardiac conductive fibers [2] showed that connective sheath displays a moderately positive reaction for type I but an intense positive reaction for type III of collagen. At 11.7 T, Qiao et al. [26] measured on ex vivo human carotid samples a MTR of $54 \pm 9 \%$ for collagen type I, whereas this value dropped to $11 \pm 6 \%$ for type III. Such a difference is thus expected to result in an increased contrast between these structures, despite both of them exhibiting MT.

\section{MT and fixation preparation}

The process of tissue fixation may also influence MTR values measured ex vivo in comparison to in vivo data, as reported by Fishbein et al. [36] at 9.4 T. They reported a slight increase of MTR (12.5\%) after fixation (with identical formalin concentrations), which cannot explain the MTR values observed in our experiments (25-44\%, see Table 2). 
Fig. 7 a Illustrative examples of immunofluorescence (IF) staining on Purkinje cells (PF) and cardiomyocyte (cardiac tissue) for collagen I (col I in red) and collagen III (col III in green) with DAPI staining in Blue. b Bar graphs illustrating quantitative results using q-PCR to evaluate expression of collagen I (col I) and collagen III (col III) in Purkinje fibers (PF) and cardiac tissue (a)
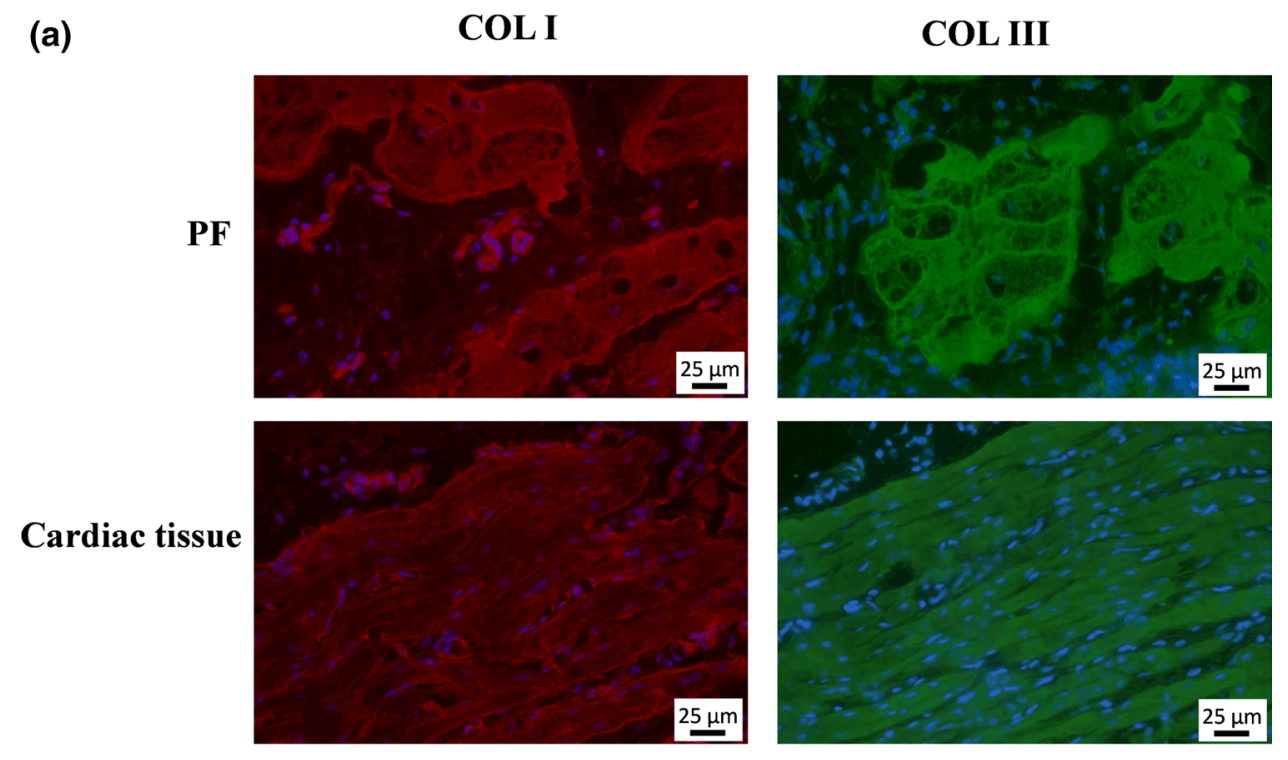

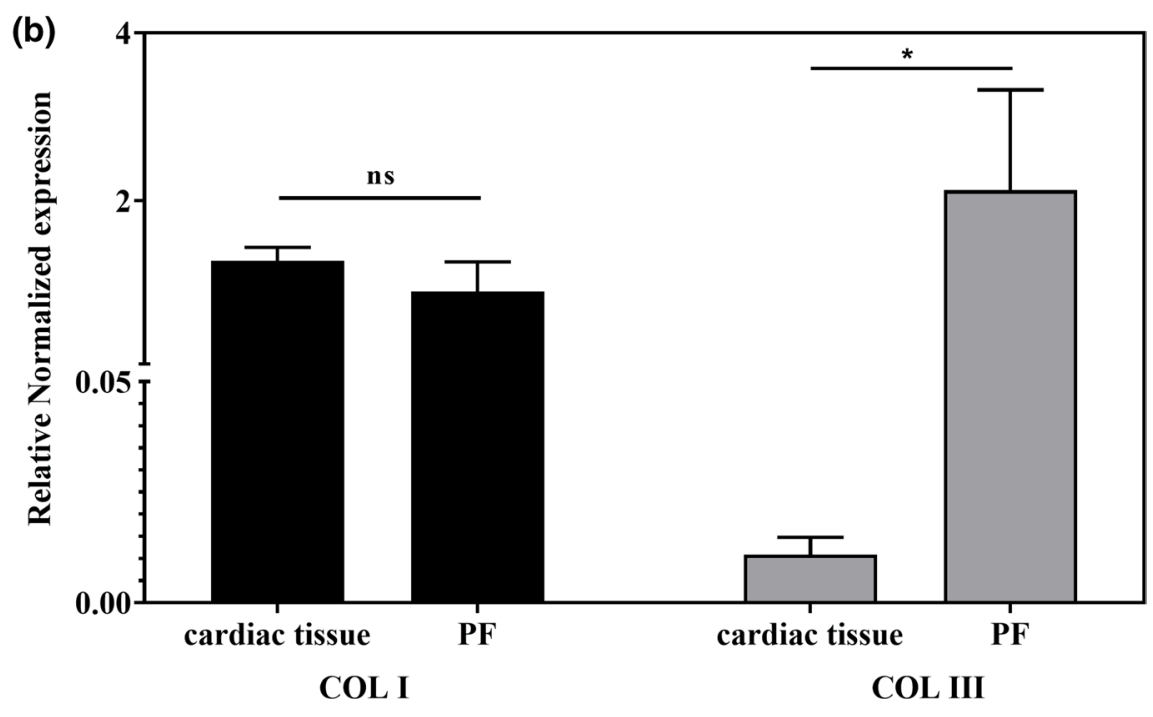

Working on ex vivo samples has an impact on $T 2$ and $T 1$ with reduced values in comparison to in vivo or unfixed post mortem samples [37]. Due to reduction of $T 1$ and $T 2$ and diffusivity, DTI acquisition at an isotropic resolution of $150 \mu \mathrm{m}$ remained challenging. The most important inconvenient with DTI is the long acquisition time to obtain a resolution in 3D of $150 \mu \mathrm{m}$ with sufficient SNR. It has been reported on fixed ex vivo sample that $T 1$ and $T 2$ contrast can be increased by a prolonged washing of the sample (wash out fixative and rehydrate the sample) before scanning in saline or phosphate-buffered saline (PBS) [38]. This preparation before MRI scanning has not been investigated here but could improve our acquisition protocol.

The increase in contrast observed in the present work using the MT technique can reasonably be attributed to different collagen types in myocardium and PF, due to different content of collagen I and collagen III demonstrated by immunofluorescence staining and q- PCR.

Histological data are in good agreement with previously published results. Pope et al. [39] performed 3D acquisition of a small piece of rabbit heart with a voxel resolution of $1 \mu \mathrm{m}^{3}$ using confocal microscopy to follow the collagen network outside and inside the muscle. Ono et al. [40] demonstrated that conductive fiber network varies between species. Indeed, in goats and sheep Purkinje strands consisting of 2-8 oval cells, they demonstrated that the mesh of the PF network varied between 200 and $1500 \mu \mathrm{m}$ using light electron microscopy. Histological images in the present study showed fibers with cardiomyocytes surrounded by collagen, which are characteristic of the free PF network, as described in the literature $[2,41]$. A transitional structure at the junction between free-running PF and myocardium can 
be observed (Fig. 6). Tranum-Jensen et al. [42] observed the same anatomic arrangement at the connection of PF to the cardiac muscle with a large PF fibers connected via thin branches to the ventricular mass.

The main limitation of this study remains the spatial resolution. Although we performed acquisition at an isotropic resolution of $150 \mu \mathrm{m}$, allowing to follow free-running PF in pig hearts ( $\sim$ size of human heart), such a voxel size remains at the limit to track intramural PFs, where a resolution of $100 \mu \mathrm{m}$ or better would be required. At this resolution, the resulting SNR would be divided by 3, leading to resulting SNR of around 10 (at the limit for interpretation) with our experimental setup. The use of higher averaging of smaller samples with dedicated instrumentation (smaller coil combined with a cryoprobe for example) in combination with further image processing is amenable to provide exploitable data at such resolution. In the present work, we chose to focus the analysis of MT parameters to increase contrast, at the cost of a compromise on image resolution. Alternatively, MT contrast could also be generated with other pulses design $[43,44]$ or acquisition techniques such as on-resonance MT [45] to reduce total acquisition duration, energy deposition, and temperature increase. This was out of the scope of the present work.

In the future, the MT methodology in ex vivo cardiac samples could also be applied to pathological hearts [45] with regions of infarct to evaluate contrast obtained between lesion sites (with collagen remodeling) and healthy regions to support realistic computational simulations and to understand electrophysiology and arrhythmias disorders [10, 39].

We demonstrated the added value of the MT technique to improve contrast between PF and cardiac muscle. Although all tissues exhibited a reduction in SNR in presence of MT, the contrast between PF and the cardiac tissue has been increased by more than $50 \%$ as compared to standard proton density weighted sequence. 3D high-resolution images at $150 \mu \mathrm{m}$ are feasible and allow to visualize the free-running $\mathrm{PF}$ and their connection within the myocardium.

Author contributions JM: drafting of manuscript and prepared figures. $\mathrm{JM}, \mathrm{AF}, \mathrm{KH}$, and $\mathrm{JN}$ : samples preparation, acquisition of data, and study conception and design. JM: programming developments and data analysis. JM, MC, and SC: histology and q-PCR experiments. JM, $\mathrm{DEH}, \mathrm{DB}, \mathrm{SC}, \mathrm{MC}, \mathrm{RW}, \mathrm{OB}$, and BQ: interpretation of data. JM, JN, $\mathrm{DEH}, \mathrm{DB}, \mathrm{SC}, \mathrm{MC}, \mathrm{RW}, \mathrm{OB}$, and BQ: critical revision.

Funding This study received financial support from the French Government as part of the Investments of the Future program managed by the National Research Agency (ANR; Grant Number ANR-10IAHU04-LIRYC). This work was also financed by the Region Nouvelle Nouvelle Aquitaine (Grant Agreement Number 2016-0127 Purkinje) and the European Research Council (FP7/2007-2013 Grant Agreement Number 322886-SYMPHONY) is acknowledged for financial support.

\section{Compliance with ethical standards}

Conflict of interest The authors declare that they have no conflict of interest.

Ethical approval Animals protocol was approved by the Animal Research Ethics Committee (Comité d'Ethique en Expérimentation Animale de Bordeaux; CEEA50) in accordance with the European rules for animal experimentation (European legislation 2010/63/UE; 2010).

Open Access This article is licensed under a Creative Commons Attribution 4.0 International License, which permits use, sharing, adaptation, distribution and reproduction in any medium or format, as long as you give appropriate credit to the original author(s) and the source, provide a link to the Creative Commons licence, and indicate if changes were made. The images or other third party material in this article are included in the article's Creative Commons licence, unless indicated otherwise in a credit line to the material. If material is not included in the article's Creative Commons licence and your intended use is not permitted by statutory regulation or exceeds the permitted use, you will need to obtain permission directly from the copyright holder. To view a copy of this licence, visit http://creativecommons.org/licenses/by/4.0/.

\section{References}

1. Eliska O (2006) Purkynje fibers of the heart conduction systemhistory and the present time. Cas Lek Cesk 145(4):329-335

2. Morita T, Shimada T, Kitamura H, Nakamura M (1991) Demonstration of connective tissue sheaths surrounding working myocardial cells and Purkinje cells of the sheep moderator band. Arch Histol Cytol 54(5):539-550

3. Boyden PA, Dun W, Robinson RB (2016) Cardiac Purkinje fibers and arrhythmias; the GK Moe award lecture 2015. Heart Rhythm 13(5):1172-1181

4. Bogun F, Good E, Reich S, Elmouchi D, Igic P, Tschopp D, Dey S, Wimmer A, Jongnarangsin K, Oral H, Chugh A, Pelosi F, Morady F (2006) Role of Purkinje fibers in post-infarction ventricular tachycardia. J Am Coll Cardiol 48(12):2500-2507

5. Haissaguerre M, Vigmond E, Stuyvers B, Hocini M, Bernus O (2016) Ventricular arrhythmias and the His-Purkinje system. Nat Rev Cardiol 13(3):155-166

6. Atkinson A, Inada S, Li J, Tellez JO, Yanni J, Sleiman R, Allah EA, Anderson RH, Zhang H, Boyett MR, Dobrzynski H (2011) Anatomical and molecular mapping of the left and right ventricular His-Purkinje conduction networks. J Mol Cell Cardiol 51(5):689-701

7. Ansari A, Ho SY, Anderson RH (1999) Distribution of the Purkinje fibres in the sheep heart. Anat Rec 254(1):92-97

8. Stephenson RS, Boyett MR, Hart G, Nikolaidou T, Cai X, Corno AF, Alphonso N, Jeffery N, Jarvis JC (2012) Contrast enhanced micro-computed tomography resolves the 3-dimensional morphology of the cardiac conduction system in mammalian hearts. PLoS ONE 7(4):e35299

9. Stephenson RS, Atkinson A, Kottas P, Perde F, Jafarzadeh F, Bateman M, Iaizzo PA, Zhao J, Zhang H, Anderson RH, Jarvis JC, Dobrzynski H (2017) High resolution 3-Dimensional imaging of the human cardiac conduction system from microanatomy to mathematical modeling. Sci Rep 7(1):7188 
10. Bordas R, Grau V, Burton RB, Hales P, Schneider JE, Gavaghan D, Kohl P, Rodriguez B (2010) Integrated approach for the study of anatomical variability in the cardiac Purkinje system: from high resolution MRI to electrophysiology simulation. Conf Proc IEEE Eng Med Biol Soc 2010:6793-6796

11. Cetingul HE, Plank G, Trayanova NA, Vidal R (2011) Estimation of local orientations in fibrous structures with applications to the Purkinje system. IEEE Trans Biomed Eng 58(6):1762-1772

12. Burton RA, Plank G, Schneider JE, Grau V, Ahammer H, Keeling SL, Lee J, Smith NP, Gavaghan D, Trayanova N, Kohl P (2006) Three-dimensional models of individual cardiac histoanatomy: tools and challenges. Ann NY Acad Sci 1080:301-319

13. Hwang M-S, Odening KE, Choi B-R, Koren G, Blackband SJ, Forder JR (2011) Non-invasive monitoring of changes in rabbit hearts with aging using MR microscocpy. J Cardiovasc Magn Reson 13(1):P361

14. Magat J, Fouillet A, Naulin J, Benoist D, Guo Y, Bernus O, Stuyvers B, Quesson B (2016) High resolution Magnetic Resonance Imaging (MRI) combined with Magnetization Transfer (MT) for the visualization of the cardiac structure: an ex vivo proof of concept. ISMRM Congress

15. Wolff SD, Balaban RS (1989) Magnetization transfer contrast (MTC) and tissue water proton relaxation in vivo. Magn Reson Med 10(1):135-144

16. Miller DH, Grossman RI, Reingold SC, McFarland HF (1998) The role of magnetic resonance techniques in understanding and managing multiple sclerosis. Brain 121(Pt 1):3-24

17. Parker DL, Buswell HR, Goodrich KC, Alexander AL, Keck N, Tsuruda JS (1995) The application of magnetization transfer to MR angiography with reduced total power. Magn Reson Med 34(2):283-286

18. Henkelman RM, Stanisz GJ, Graham SJ (2001) Magnetization transfer in MRI: a review. NMR Biomed 14(2):57-64

19. Fuchs BC, Wang H, Yang Y, Wei L, Polasek M, Schuhle DT, Lauwers GY, Parkar A, Sinskey AJ, Tanabe KK, Caravan P (2013) Molecular MRI of collagen to diagnose and stage liver fibrosis. J Hepatol 59(5):992-998

20. Jiang K, Ferguson CM, Ebrahimi B, Tang H, Kline TL, Burningham TA, Mishra PK, Grande JP, Macura SI, Lerman LO (2017) Noninvasive assessment of renal fibrosis with Magnetization Transfer MR imaging: validation and evaluation in murine renal artery stenosis. Radiology 283(1):77-86

21. Jiang K, Ferguson CM, Woollard JR, Zhu X, Lerman LO (2017) Magnetization Transfer magnetic resonance imaging noninvasively detects renal fibrosis in swine atherosclerotic renal artery stenosis at 3.0 T. Invest Radiol 52(11):686-692

22. Adler J, Swanson SD, Schmiedlin-Ren P, Higgins PD, Golembeski CP, Polydorides AD, McKenna BJ, Hussain HK, Verrot TM, Zimmermann EM (2011) Magnetization transfer helps detect intestinal fibrosis in an animal model of Crohn disease. Radiology 259(1):127-135

23. Karina L, Radhouene N, Mukherjee RK, John W, Alkystis P, Reza R, Claudia P, Sébastien R, René B (2020) Contrast-free highresolution $3 \mathrm{D}$ magnetization transfer imaging for simultaneous myocardial scar and cardiac vein visualization. Magnet Reso Materials Phys, Biol Med 33(5):627-640

24. Weiss CR, Aletras AH, London JF, Taylor JL, Epstein FH, Wassmuth R, Balaban RS, Arai AE (2003) Stunned, infarcted, and normal myocardium in dogs: simultaneous differentiation by using gadolinium-enhanced cine MR imaging with magnetization transfer contrast. Radiology 226(3):723-730

25. de Souza RR (2002) Aging of myocardial collagen. Biogerontology 3(6):325-335

26. Qiao Y, Hallock KJ, Hamilton JA (2011) Magnetization transfer magnetic resonance of human atherosclerotic plaques ex vivo detects areas of high protein density. J Cardiovasc Magn Reson $13: 73$

27. Gilbert SH, Benoist D, Benson AP, White E, Tanner SF, Holden AV, Dobrzynski H, Bernus O, Radjenovic A (2012) Visualization and quantification of whole rat heart laminar structure using highspatial resolution contrast-enhanced MRI. Am J Physiol Heart Circ Physiol 302(1):H287-298

28. Cleary JO, Price AN, Thomas DL, Scambler PJ, Kyriakopoulou V, McCue K, Schneider JE, Ordidge RJ, Lythgoe MF (2009) Cardiac phenotyping in ex vivo murine embryos using microMRI. NMR Biomed 22(8):857-866

29. Hales PW, Burton RA, Bollensdorff C, Mason F, Bishop M, Gavaghan D, Kohl P, Schneider JE (2011) Progressive changes in T(1), $\mathrm{T}(2)$ and left-ventricular histo-architecture in the fixed and embedded rat heart. NMR Biomed 24(7):836-843

30. Gilbert S, Trew M, Smaill B, Radjenivic A, Bernus O (2011) Measurement of myocardial structure: 3D structure tensor analysis of high resoluion MRI quantitatively compared to DT-MRI. Paper presented at the STACOM 2012. Lecture Notes in Computer Science. Berlin, Heidelberg

31. Bernus O, Radjenovic A, Trew M, LeGrice I, Sands G, Magee D, Smaill B, Gilbert S (2015) Comparison of diffusion tensor imaging by cardiovascular magnetic resonance and gadolinium enhanced 3D image intensity approaches to investigation of structural anisotropy in explanted rat hearts. J Cardiovasc Magn Reson $17: 31$

32. Jones RA, Haraldseth O, Schjott J, Brurok H, Jynge P, Oksendal AN, Rinck PA (1993) Effect of Gd-DTPA-BMA on magnetization transfer: application to rapid imaging of cardiac ischemia. J Magn Reson Imaging 3(1):31-39

33. Kurki TJ, Niemi PT, Lundbom N (1992) Gadolinium-enhanced magnetization transfer contrast imaging of intracranial tumors. J Magn Reson Imaging 2(4):401-406

34. Mathews VP, Elster AD, King JC, Ulmer JL, Hamilton CA, Strottmann JM (1995) Combined effects of magnetization transfer and gadolinium in cranial MR imaging and MR angiography. AJR Am J Roentgenol 164(1):169-172

35. Parker DL, Smith V, Sheldon P, Crooks LE, Fussell L (1983) Temperature distribution measurements in two-dimensional NMR imaging. Med Phys 10(3):321-325

36. Fishbein KW, Gluzband YA, Kaku M, Ambia-Sobhan H, Shapses SA, Yamauchi M, Spencer RG (2007) Effects of formalin fixation and collagen cross-linking on $T 2$ and magnetization transfer in bovine nasal cartilage. Magn Reson Med 57(6):1000-1011

37. Roebroeck A, Miller KL, Aggarwal M (2019) Ex vivo diffusion MRI of the human brain: technical challenges and recent advances. NMR Biomed 32(4):e3941

38. Shepherd TM, Thelwall PE, Stanisz GJ, Blackband SJ (2009) Aldehyde fixative solutions alter the water relaxation and diffusion properties of nervous tissue. Magn Reson Med 62(1):26-34

39. Pope AJ, Sands GB, Smaill BH, LeGrice IJ (2008) Three-dimensional transmural organization of perimysial collagen in the heart. Am J Physiol Heart Circ Physiol 295(3):H1243-H1252

40. Ono N, Yamaguchi T, Ishikawa H, Arakawa M, Takahashi N, Saikawa T, Shimada T (2009) Morphological varieties of the Purkinje fiber network in mammalian hearts, as revealed by light and electron microscopy. Arch Histol Cytol 72(3):139-149

41. Walton RD, Pashaei A, Martinez ME, Constantin M, Duchateau J, Bear L, Cros C, Pascarel-Auclerc C, Guo Y, Benoist D, Dubes V, Faye NR, Chaigne S, Dupuis S, Detaille D, Pourtau L, Pasdois P, Brette F, Rogier J, Labrousse L, Hocini M, Vigmond EJ, Haissaguerre M, Bernus O (2018) Compartmentalized structure of the moderator band provides a unique substrate for macroreentrant ventricular tachycardia. Circ Arrhythm Electrophysiol 11(8):e005913 
42. Tranum-Jensen J, Wilde AA, Vermeulen JT, Janse MJ (1991) Morphology of electrophysiologically identified junctions between Purkinje fibers and ventricular muscle in rabbit and pig hearts. Circ Res 69(2):429-437

43. Pachot-Clouard M, Darrasse L (1995) Optimization of $T 2$-selective binomial pulses for magnetization transfer. Magn Reson Med 34(3):462-469

44. Varma G, Duhamel G, de Bazelaire C, Alsop DC (2015) Magnetization transfer from inhomogeneously broadened lines: a potential marker for myelin. Magn Reson Med 73(2):614-622
45. Weber OM, Speier P, Scheffler K, Bieri O (2009) Assessment of magnetization transfer effects in myocardial tissue using balanced steady-state free precession (bSSFP) cine MRI. Magn Reson Med 62(3):699-705

Publisher's Note Springer Nature remains neutral with regard to jurisdictional claims in published maps and institutional affiliations. 